\title{
Kimura's Disease With Recurrent Bilateral Lacrimal Gland Involvement in a Male Japanese Child Successfully Treated With Cyclosporine A
}

Keisuke Sugimoto ( $\nabla$ ksugimo@med.kindai.ac.jp )

Kindai University Faculty of Medicine https://orcid.org/0000-0002-0978-7511

\section{Takuji Enya}

Kindai University Faculty of Medicine Hospital: Kinki Daigaku Igakubu Daigaku Byoin

\section{Yuichi Morimoto}

Kindai University Faculty of Medicine Hospital: Kinki Daigaku Igakubu Daigaku Byoin

\section{Rina Oshima}

Kindai University Faculty of Medicine Hospital: Kinki Daigaku Igakubu Daigaku Byoin

\section{Kohei Miyazaki}

Kindai University Faculty of Medicine Hospital: Kinki Daigaku Igakubu Daigaku Byoin

\section{Mitsuru Okada}

Kindai University Faculty of Medicine Hospital: Kinki Daigaku Igakubu Daigaku Byoin

\section{Case report}

Keywords: Kimura's disease, cyclosporine, children, lacrimal gland

Posted Date: February 5th, 2021

DOl: https://doi.org/10.21203/rs.3.rs-183210/v1

License: (9) (i) This work is licensed under a Creative Commons Attribution 4.0 International License. Read Full License 


\section{Abstract}

\section{Background}

Kimura's disease (KD) is a rare chronic inflammatory disease of unknown etiology. Clinically, KD is characterized by nodular subcutaneous masses, typically localized to the neck and head. Involvement of the lacrimal glands and limbs is uncommon and seldom reported.

\section{Case presentation}

We report a case of a 4-year-old Japanese boy presenting with bilateral upper eyelid swelling with nodular subcutaneous lesions and peripheral eosinophilia. Based on clinical, histopathological, and laboratory findings, the patient was diagnosed with KD. An itchy subcutaneous mass on the left arm developed at the age of 14 years. Treatment with steroids was effective, but the masses relapsed within a few months as the steroids were tapered after the patient developed side effects. Treatment with cyclosporine A was initiated, which led to the improvement of clinical features and serial levels of cytokines.

\section{Conclusions}

We report a rare case of $\mathrm{KD}$ with a peculiar clinical presentation. The patient responded well to treatment with CsA.

\section{Background}

Kimura's disease (KD) is an inflammatory condition that classically manifests as painless subcutaneous nodules on the head and neck and characteristically occurs in young Asian males of any age [1, 2]. However, ocular involvement in KD is uncommon, especially in children [3]. Additionally, only a small number of KD cases that include upper limb lesions have been reported. The pathophysiology of KD remains unknown but allergic reactions (caused by parasites, viruses, fungi, or toxins), atopy, and autoimmunity are considered possible risk factors that may cause activation of the cytokine pathway that is implicated in $\mathrm{KD}[2,4,5]$. Histologically, KD is characterized by lymphoid follicles with germinal centers that are distinguished by an eosinophilic infiltrate and varying degrees of fibrosis $[4,5]$.

Here, we describe a unique case of KD in a Japanese boy. The case exhibited bilateral orbital involvement and was successfully treated with cyclosporine A (CsA). To the best of our knowledge, this is the youngest case of KD with involvement of the bilateral lacrimal gland and the left arm reported in the English literature.

\section{Case Presentation}

A 4-year-old Japanese boy presented with bilateral upper eyelid swelling and discomfort. The swelling was painless, but he complained of itchy eyelids. Ophthalmic examination revealed bilateral upper eyelid edema (Fig. 1). No lymphadenopathy of the head or neck was observed. The patient had no medical 
history of atopic dermatitis, but local reactions to insect bites and vaccinations were excessive. There was no family history of autoimmune renal disease.

Urinalysis results were normal; blood profiling showed a white blood cell count of $8,200 / \mathrm{mL}$ with a differential neutrophil count of $13 \%$, a lymphocyte count of $55.5 \%$, and a markedly increased eosinophil count of $28.5 \%$. A peripheral smear revealed no abnormalities. The CD4/CD8 ratio was normal (1.46). Most laboratory blood examinations included electrolytes, serum creatinine, and blood urea nitrogen quantitation. The patient had an elevated aspartate aminotransferase level (34 IU/L; normal range, 14-20 $\mathrm{IU} / \mathrm{L}$ ) and alanine aminotransferase activity within normal range (16 IU/L; normal range, 10-40 IU/L). Lactate dehydrogenase and creatinine kinase activities were normal. The patient's C-reactive protein level was $0.045 \mathrm{mg} / \mathrm{dL}$, and he had an erythrocyte sedimentation rate of $9 \mathrm{~mm} / \mathrm{h}$. His $\lg \mathrm{G}, \lg \mathrm{A}, \lg \mathrm{D}, \lg \mathrm{M}$, and IgG4 levels were normal (IgG, 1,173 mg/dL; IgA, 162 mg/dL; IgD, 9.9 mg/dL; lgM 206 mg/dL; and IgG4 9.7 $\mathrm{mg} / \mathrm{dL})$. The patient had a highly elevated serum IgE level (14,351 IU/mL; reference range: $0-295 \mathrm{IU} / \mathrm{mL})$. Serologic analyses for antinuclear antibody and MPO-ANCA were negative and serum complement components $\mathrm{C} 3, \mathrm{C} 4$, and total complement activity were normal. Chest radiography results were also normal, though magnetic resonance imaging (MRI) showed bilateral lacrimal gland enlargement without the appearance of mass lesions in the orbit and adjacent bony erosion (Fig. 2).

Mikulicz disease was initially suspected, despite the normal IgG4 level $(9.7 \mathrm{mg} / \mathrm{dL})$. Based on this presumptive diagnosis, pranlukast hydrate was administered for 2 years. However, follow-up treatment was suspended for 4 years because symptoms did not improve. An excisional biopsy via anterior orbitotomy was performed at the age of 10 years and revealed eosinophilic hyperplastic lymphogranuloma involving the lacrimal gland (Fig. 3). A mixture of CD3- and CD5-positive T-cells was found within the interfollicular regions, and CD20-positive B-cells were observed within the follicles. A relatively less number of IgG- and IgG4-positive plasma cells were also observed. There was no evidence of either vasculitis or prominent vascular endothelial cell proliferation.

Based on these findings, IgG4-related diseases, including Mikulicz disease, were excluded. Oral prednisolone was initiated at $1 \mathrm{mg} / \mathrm{kg} /$ day based on a diagnosis of lymphoproliferative disorder. The patient's symptoms, including eyelid edema, discomfort, and eosinophilia, had improved dramatically at the 1-month follow-up, but symptoms recurred as prednisolone was tapered. Subsequently, a combinatorial therapy with mizoribine was initiated to mitigate the side effects of steroids. However, swelling of the bilateral eyelids worsened, and an itchy subcutaneous mass developed on the left arm. MRI examination of the right arm revealed serpiginous subcutaneous lesions in the medial aspect of the left distal arm (Fig. 4). The masses of the bilateral eyelids were resected when the patient was 14 years old. Postoperative pathological examination showed hyperplastic lymphoid follicles embedded in fibroconnective tissue and multinuclear cells of Whartin-Finkeldey type, indicative of KD (Fig. 5). Immunohistochemical analysis of the cells revealed negative staining for IgG and IgG4. The serum IL4level was elevated ( $500 \mathrm{pg} / \mathrm{mL}$; reference range: $<6.0 \mathrm{pg} / \mathrm{mL}$ ), but IL-5, IL-6, and interferon $y$ levels were normal. Serum sIL-2R levels were maintained in the reference range throughout the clinical course. The diagnosis of KD was confirmed based on these histological findings, along with markedly high serum IgE 
levels and peripheral blood eosinophilia. Treatment with CsA at an initial dose of $2.5 \mathrm{mg} / \mathrm{kg} / \mathrm{day}$ was started and maintained at a trough level of around $50 \mathrm{ng} / \mathrm{ml}$. After initiation of oral CsA, eosinophilia and serum IgE levels decreased gradually, although the IL-4 levels remained high. The clinical symptoms were stable without bilateral upper eyelid swelling and subcutaneous mass on the left arm with $4 \mathrm{mg} / \mathrm{kg}$ per day of CsA therapy.

\section{Discussion And Conclusions}

KD patients almost always have marked eosinophilia and elevated serum IgE levels. Some studies have reported that blood eosinophil counts in KD patients are correlated with lesion size, that is, larger lesions are associated with higher eosinophil count [6]. Common symptoms of KD include the head and neck region, and CT and MRI show a wide range of lesions and multiple enlarged lymph nodes [7]. Orbital involvement, including eyelid swelling, has also been reported [8-12], but is uncommon. Furthermore, most KD cases with orbital involvement were adults from Asian countries, and, to our knowledge, the case reported here is the youngest case of KD with orbital involvement. Additionally, our patient developed a lesion in his left arm as a result of his condition. The patient opted for surgical excision of the left arm mass because, other than itchiness, the lesion was asymptomatic. The involvement of the arm of a KD patient is rare but not unique, as Lam et al. have previously reported a case of KD affecting the anterolateral aspect of the upper arm [13]. In that earlier case, the skin lesion specimen did not show characteristic findings of KD; however, this could be attributed to the fact that only surface tissue was obtained by biopsy. However, similarities in clinical features of our case and that reported by Lam et al., i.e. an itchy left arm mass and similar MRI findings, suggest involvement of KD.

Our patient exhibited elevated eosinophil and IgE levels as the swelling of the eyelids worsened. Unfortunately, definitive diagnosis was delayed, although a related disease was initially suspected, which delayed initiation of appropriate therapeutic intervention. Clinically, the differential diagnosis of KD may include IgG4-related diseases, Hodgkin's lymphoma, Castleman's disease, Langerhans cell histiocytosis, angiolymphoid hyperplasia with eosinophilia (ALHE), drug reactions, and allergies. These histological features may overlap with those of idiopathic orbital inflammation $[8,12]$ or ALHE $[1,2]$. Notably, some cases of KD occurring in the left lacrimal gland with increased serum levels of IgG4, mimicking IgG4related disease, have been reported $[14,15]$. In the current case, we made a differential diagnosis of IgG4related disease, despite normal IgG4 levels and lack of IgG4 expression in the lacrimal gland specimen. In the literature, the most common misdiagnosis of KD is ALHE, although patients with ALHE have normal IgE levels and no renal complications $[4,10,16,17]$. In our case, there were no signs of renal disorders. Histologically, Warthin-Finkeldey cells were not observed in the first biopsy, but were detected in the lacrimal gland tissue following the second operation. A Warthin-Finkeldey cell is a giant multinucleate cell found in hyperplastic lymph nodes early in the course of measles and also in HIV-infected individuals, neoplastic and non-neoplastic lymph node disorders, and in KD [18-20]. Collectively, the clinical features and other histopathological findings ultimately indicated a definitive diagnosis of KD. 
No standard treatment for KD has been established. Suggested therapeutic options include oral or intralesional corticosteroids, surgical removal, and radiotherapy. In most cases, oral corticosteroids are effective as an initial treatment, but relapses have been frequently reported after surgery or withdrawal of oral steroids [21, 22]. Other agents, including CsA, have been reported to induce and/or maintain remission, and there are several reports of treatment with cyclosporine [21-23]. Cyclosporine acts to reduce IL-2 synthesis, resulting in inhibition of T-lymphocyte proliferation and attenuation of the immune response. Sato et al. reported an 11-year-old boy with cervical lymphadenopathy who was successfully treated with steroids and CsA [22]. Similarly, Shin et al. reported an 8-year-old boy with right upper eyelid swelling who was diagnosed with KD and was effectively treated with steroids, CsA, and azathioprine [24].

Nephrotic syndrome (NS) has been reported as a complication of KD [21, 25, 26]. Among 11 Japanese KD patients with NS, 2 were treated with CsA [4]. Katagiri et al. explained that mRNA levels of cytokines and eosinophils were suppressed when the blood concentration of CsA was $>75 \mathrm{ng} / \mathrm{mL}$ in an adult patient with KD [27]. Marked eosinophilia and elevated serum IgE were associated with regulation of Th2 cell cytokines, such as IL-4, IL-5, IL-13, and interferon-g mRNA [27, 28], and CsA efficacy may be improved by the suppression of these activities in KD [27]. Although sIL-2R levels were normal in the current case, IL-4, peripheral eosinophil counts, and IgE levels were elevated. As in previous reports, administration of CsA improved clinical features, including laboratory findings, and was maintained. NS complications during the clinical course of KD with a lacrimal gland mass have been reported previously [25]. Given the high rate of recurrence and reported association with lymphoma [29], careful long-term follow-up in is recommended for our case.

In summary, we have described a case of KD with rare or possibly unique clinical manifestations in a patient that would not normally be considered to be at risk of KD. Indeed, the incongruence with the clinical presentations initially determined in this case led to inexact diagnoses that delayed initiation of effective therapeutic intervention for several years. The details of this case provide valuable information about the range of clinical presentations that can be associated with KD, which can aid timely diagnosis and increase the efficacy of therapies.

\section{Abbreviations}

ALHE: Angiolymphoid hyperplasia with eosinophilia

CsA: Cyclosporine A

KD: Kimura's disease

MRI: Magnetic resonance imaging

NS: Nephrotic syndrome 


\section{Declarations}

Ethics approval and consent to participate

Consent was obtained from a parent or guardian on behalf of any participant under the age of 16 years. The study was conducted in accordance with the ethical principles of the Declaration of Helsinki.

\section{Consent for publication}

Written informed consent was obtained from the patient and her parents for the publication of this case report and accompanying images.

Availability of data and materials

Not applicable.

Competing interests

The authors declare that they have no competing interests.

\section{Funding}

The authors declare that no financial assistance was received for this study.

\section{Authors' contributions}

KS wrote the manuscript and reviewed the relevant literature. $\mathrm{RO}$ and $\mathrm{YM}$ assisted in the collection and interpretation of the clinical and laboratory data. TE and KM performed the histological examinations. MO critically reviewed the manuscript and supervised the study. All authors read and approved the final manuscript.

\section{Acknowledgments}

We would like to express our sincere appreciation to the patients' parents for their permission granted to publish this case. The authors would like to thank Editage for the English language review.

\section{References}

1. Kuo TT, Shih LY, Chan HL. Kimura's disease. Involvement of regional lymph nodes and distinction from angiolymphoid hyperplasia with eosinophilia. Am J Surg Pathol. 1988;12(11):843-54

2. Urabe A, Tsuneyoshi M, Enjoji M. Epithelioid hemangioma versus Kimura's disease: a comparative clinicopathologic study. Am J Surg Pathol. 1987;11(10):758-66

3. Thomas J, Jayachandran NV, Chandrasekhara PK, Rajasekhar L, Narsimulu G. Kimura's disease-an unusual cause of lymphadenopathy in children. Clin Rheumatol. 2008;27(5):675-7 
4. Chen H, Thompson LD, Aguilera NS, Abbondanzo SL. Kimura disease: A clinicopathologic study of 21 cases. Am J Surg Pathol. 2004;28(4):505-13

5. Abuel-Haija M, Hurford MT. Kimura disease. Arch Pathol Lab Med. 2007;131(4):650-1

6. Sakamoto M, Komura A, Nishimura S. Hematoserological analysis of Kimura's disease for optimal treatment. Otolaryngol Head Neck Surg. 2005;132(1):159-60

7. Li TJ, Chen XM, Wang SZ, Fan MW, Semba I, Kitano M. Kimura's disease: a clinicopathologic study of 54 Chinese patients. Oral Surg Oral Med Oral Pathol Oral Radiol Endod. 1996;82(5):549-55

8. Yuen SJ, Rubin PA. Idiopathic orbital inflammation: ocular mechanism and clinicopathology. Ophthalmol Clin North Am. 2002;15(1):121-6

9. Kennedy SM, Pitts JF, Lee WR, Gibbons DC. Bilateral Kimura's disease of the eyelids. Br J Ophthalmol. 1992;76(12):755-7

10. Sánchez-Acosta A, Moreno-Arredondo D, Rubio-Solornio RI, Rodríguez-Martínez HA, Rodríguez-Reyes AA. Angiolymphoid hyperplasia with eosinophilia of the lacrimal gland: a case report. Orbit. 2008;27(3):195-8

11. Kodama T, Kawamoto K. Kimura's disease of the lacrimal gland. Acta Ophthalmol Scand. 1998;76(3):374-7

12. Buggage RR, Spraul CW, Wojno TH, Grossniklaus HE. Kimura disease of the orbit and ocular adnexa. Surv Ophthalmol. 1999;44(1):79-91

13. Lam AC, Au Yeung RK, Lau VW. A rare disease in an atypical location-Kimura's disease of the upper extremity. Skelet Radiol. 2015;44(12):1833-7

14. Mancera N, Bajric J, Margo CE. IgG4-rich reactive lymphoid hyperplasia of the lacrimal gland. Orbit. 2020;39(4):285-8

15. Li J, GeX, Ma J, Li M, Li J. Kimura's disease of the lacrimal gland mimicking IgG4-related orbital disease. BMC Ophthalmol. 2014;14(14):158

16. Zhou LL, Zhang RZ. Misdiagnosis of uncommon presentation of angiolymphoid hyperplasia with Eosinophilia. Clin Case Rep. 2019;7(7):1273-5

17. Iguchi Y, Inoue T, Shimono M, Yamamura T, Shigematsu T, Takahashi S. Kimura's disease and its relation to angiolymphoid hyperplasia with eosinophilia: report of three cases and review of the literature. J Oral Pathol. 1986;15(3):132-7

18. Nozawa Y, Ono N, Abe M, Sakuma H, Wakasa H. An immunohistochemical study of WarthinFinkeldey cells in measles. Pathol Int. 1994;44(6):442-7

19. Kjeldsberg CR, Kim H. Polykaryocytes resembling Warthin-Finkeldey giant cells in reactive and neoplastic lymphoid disorders. Hum Pathol. 1981;12(3):267-72

20. Battaglione V, le Tourneau A, Soler C, Deville A, Michiels JF, Hofman P. Kimura's disease of the lymph node. Histology, immunohistochemistry, and differential diagnosis. Ann Pathol. 1998;18(6):492-6

21. Nakahara C, Wada T, Kusakari J, Kanemoto K, Kinugasa H, Sibasaki M, et al. Steroid sensitive nephrotic syndrome associated with Kimura disease. Pediatr Nephrol. 2000;14(6):482-5 
22. Satoshi S, Kawashima H, Kuboshima S, Watanabe K, Kashiwagi Y, Takekuma K, et al. Combined treatment of steroids and cyclosporine in Kimura disease. Pediatrics. 2006;118:921-3

23. Kaneko K, Aoki M, Hattori S, Sato M, Kawana S. Successful treatment of Kimura's disease with cyclosporine. J Am Acad Dermatol. 1999;41 (5 Pt 2):893-4

24. Shin ST, Yang YH, Chiang BL. Recurrent Kimura's disease: report of one case. Acta Paediatr Taiwan. 2007;48(3):149-51

25. Rajpoot DK, Pahl M, Clark J. Nephrotic syndrome associated with Kimura disease. Pediatr Nephrol. 2000;14(6):486-8

26. Matsuo T, Tanaka T, Kinomura M. Nephrotic syndrome during the tapering of oral steroids after pathological diagnosis of Kimura disease from a lacrimal gland mass: case report and review of 10 Japanese patients. J Clin Exp Hematop. 2017;57(3):147-52

27. Katagiri K, Itami S, Hatano Y, Yamaguchi T, Takayasu S. In vivo expression of IL-4, IL-5, IL-13 and IFNgamma mRNAs in peripheral blood mononuclear cells and effect of cyclosporin $A$ in a patient with Kimura's disease. Br J Dermatol. 1997;137(6):972-7

28. Kimura Y, Pawankar R, Aoki M, Niimi Y, Kawana S. Mast cells and T cells in Kimura's disease express increased levels of interleukin-4, interleukin-5, eotaxin and RANTES. Clin Exp Allergy. 2002;32(12):1787-93

29. Zhang $X$, Jiao Y. The clinicopathological characteristics of Kimura disease in Chinese patients. Clin Rheumatol. 2019;38(12):3661-7

\section{Figures}

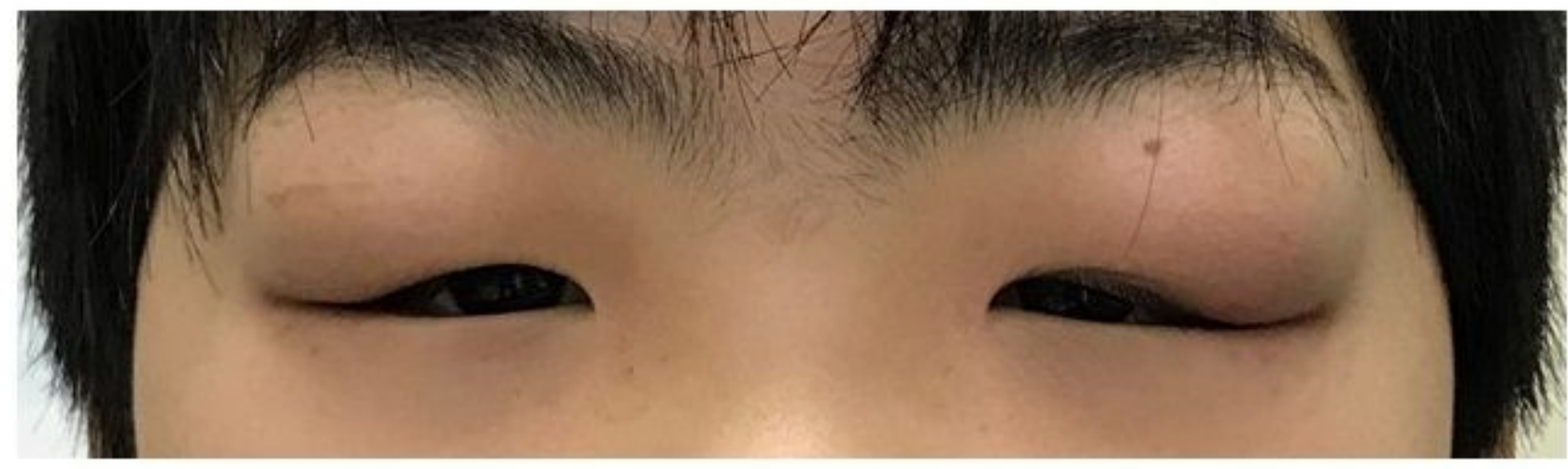

\section{Figure 1}




\section{Figure 1}

Clinical photograph of patient The patient presented with bilateral swollen eyelids
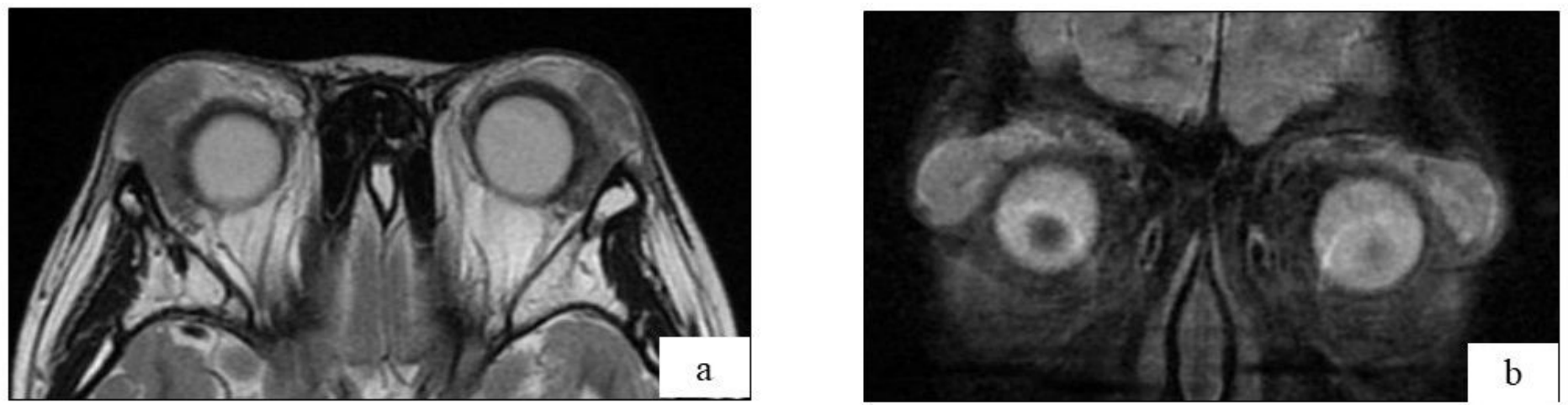

\section{Figure 2}

\section{Figure 2}

Magnetic resonance imaging ( $a, b)$ T2-weighted axial and coronal MRI demonstrated presence of an enhanced lesion of the bilateral lacrimal gland
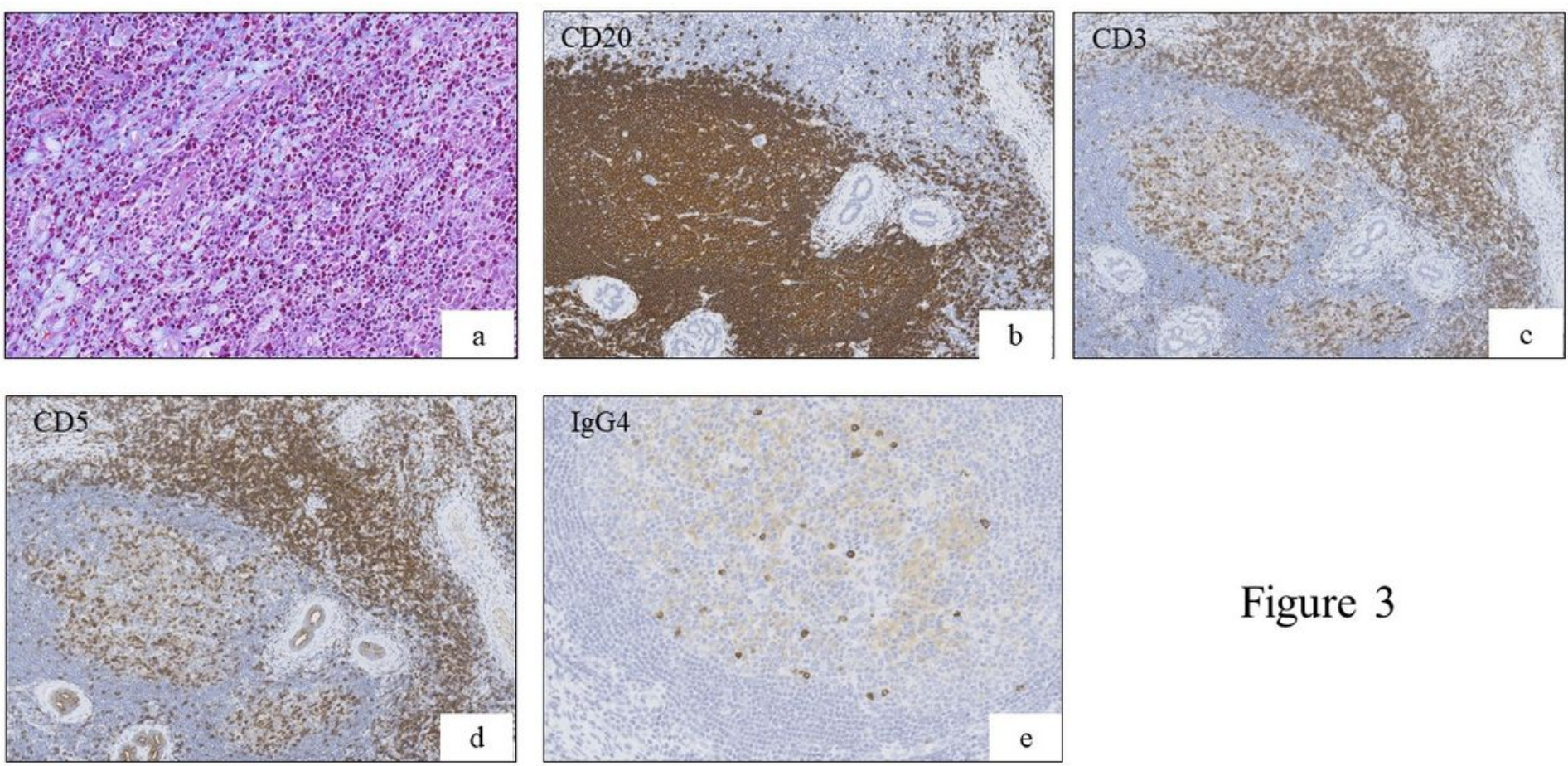

Figure 3

Figure 3 
Pathological findings of lacrimal gland (first biopsy) (a) Diffuse infiltration of the lacrimal gland by eosinophilic hyperplastic lymphogranuloma with fibrosis (Masson's trichrome staining, 200X). (b, c, d) CD20-positive B-cells within follicles; CD3- and CD5-positive T-cells were present within the interfollicular regions (100X). (e) A small proportion of IgG4-positive plasma cells were observed (200X)
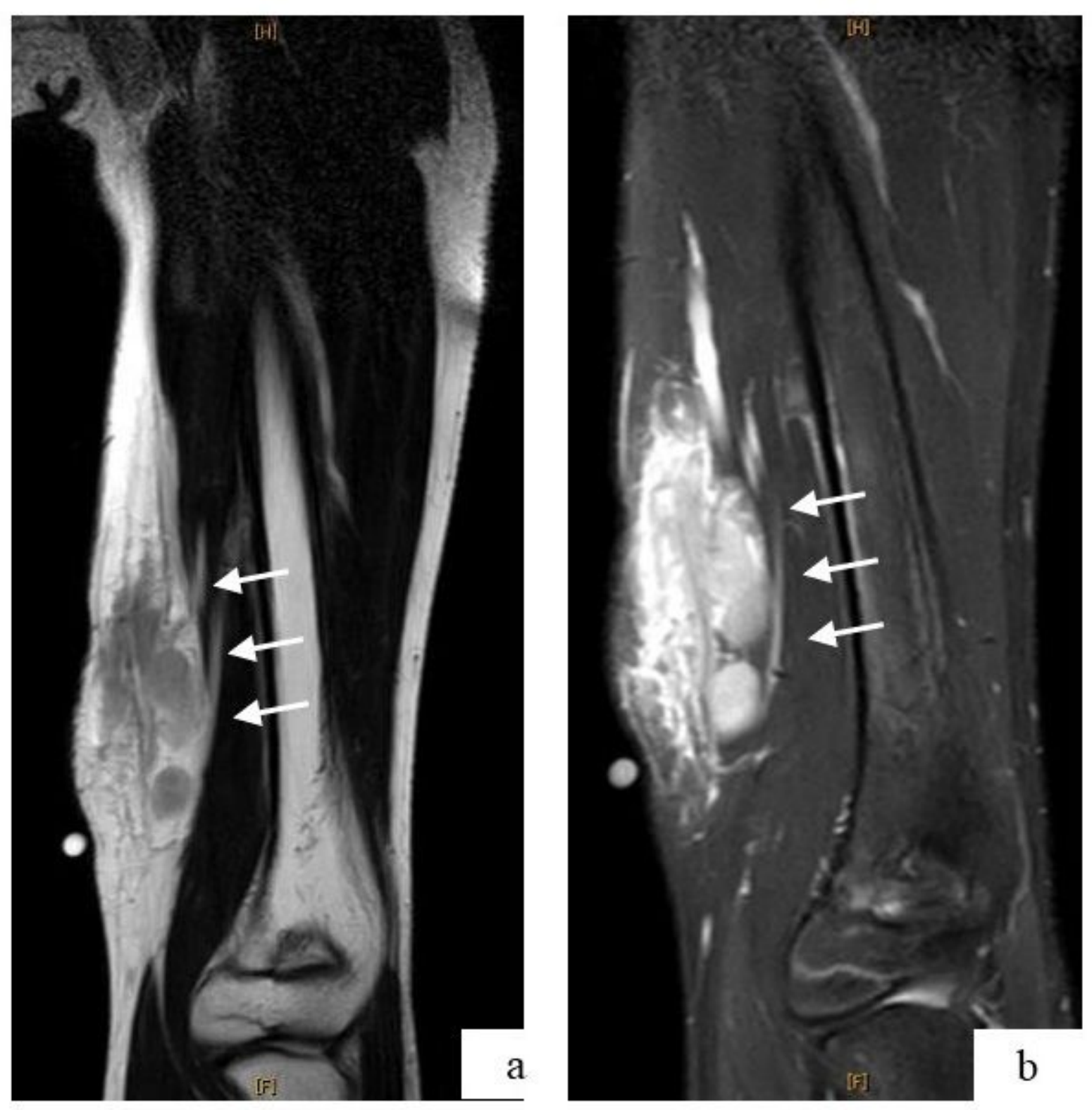

\section{Figure 4}

\section{Figure 4}

Magnetic resonance imaging of the right proximal arm (a) T2-weighted coronal MR image reveals presence of a subcutaneous lesion in the medial aspect of the left distal arm (white arrow). (b) Fat suppression T2-weighted coronal MR image shows presence of a lesion of complex intermediate signal intensity (white arrow) 

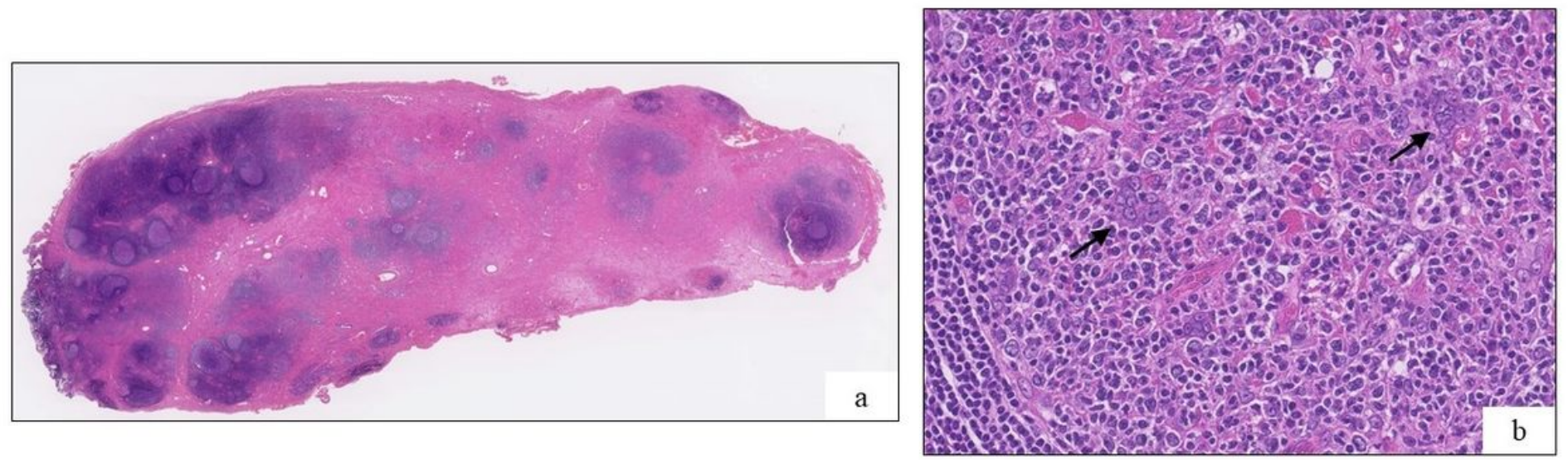

Figure 5

\section{Figure 5}

Pathological findings of lacrimal gland (second operation) (a) Hyperplastic lymphoid follicles embedded in fibroconnective tissue (Hematoxylin and eosin staining, 5X). (b) Warthin-Finkeldey cells are observed within the follicular mantle zones (black arrow) (hematoxylin and eosin staining, 300X)

\section{Supplementary Files}

This is a list of supplementary files associated with this preprint. Click to download.

- 20210129CARE.pdf 